\title{
Intelligente Zielführung elektrischer Fahrzeuge mit Brennstoffzelle als Range Extender in vernetzten Verkehrssystemen
}

\author{
Sören Scherler*, Xiaobo Liu-Henke \\ Ostfalia Hochschule für angewandte Wissenschaften, Fakultät Maschinenbau, Institut für Mechatronik (IMEC), \\ Salzdahlumer Str. 46/48, 38302 Wolfenbüttel, Deutschland; *so.scherler@ostfalia.de
}

\begin{abstract}
Im vorliegenden Beitrag wird die intelligente Zielführung elektrischer Fahrzeuge mit Brennstoffzelle als Range Extender in vernetzten Verkehrssystemen behandelt. Diese Zielführung ermittelt eine Route, welche entweder bzgl. Energieverbrauch, Zeit, Distanz oder einer Gewichtung dieser Zielkriterien optimal ist. Des Weiteren wird eine Schnittstelle zu Informationen aus der V2X-Kommunikation vorgesehen, sodass aktuelle Verkehrsdaten und auch Informationen über die Lade- und Tankinfrastruktur berücksichtigt werden können. Diese Informationen werden zur optimalen Planung von Lade- und Tankstopps auf Fahrten genutzt, für welche die in der Batterie und/oder dem Wasserstofftank gespeicherte Energie nicht ausreicht. Diese Stopps werden bzgl. der zuvor genannten Kriterien geplant.
\end{abstract}

\section{Einleitung}

Im Teilprojekt Intelligente Elektrofahrzeuge mit Range Extender in Verkehrssystemen mit Fahrzeug 4.0 des vom Niedersächsischen Ministeriums für Wissenschaft und Kultur sowie der VolkswagenStiftung geförderten Verbundprojekts Zukünftige Fahrzeugtechnologien im Open Region Lab werden automatisierte Elektrofahrzeuge mit Brennstoffzellen als Range Extender untersucht. Ziel des Teilprojekts ist die Erzielung eines zeit- und energieoptimierten, prädiktiven Fahrbetriebs in vernetzten Verkehrssystemen. Um dieses Ziel zu erreichen, werden drei Optimierungspotentiale in der Zielführung (,Welche Route ist optimal?“), der Bahnplanung (,Welche Fahrzeugführung ist auf der gewählten Route optimal?") und dem Energiemanagement (,Welche Verteilung der zur Erfüllung der Fahraufgabe notwendigen Leistung auf Batterie und Brennstoffzelle ist optimal?") fokussiert.
Die Zielführung eines elektrischen Fahrzeugs mit Batterie und Brennstoffzelle als Range Extender ist insofern eine Herausforderung, als dass je nach Betriebsmodus (Batteriebetrieb, Brennstoffzellenbetrieb, Leistungsverteilter Betrieb beider Energiespeicher) bei nicht ausreichender Reichweite zum Ziel unterschiedliche Ladeund Tankinfrastruktur berücksichtigt werden muss. Insbesondere der leistungsverteilte Betrieb, welcher aufgrund einer optimalen Energiebereitstellung durch Batterie und Brennstoffzelle am effizientesten ist, erfordert eine gute Planung der Lade- und Tankstopps, da sowohl Batterie als auch Brennstoffzelle zu jedem Zeitpunkt über ausreichend Energie verfügen müssen, um das Wirkungsgradoptimum erzielen zu können.

Die Problemstellung liegt in der Planung der Route unter Berücksichtigung notwendiger Lade- und Tankstopps bei minimal möglicher Erhöhung der Fahrtdauer, weshalb in diesem Beitrag die modellbasierte Entwicklung einer intelligenten Zielführung elektrischer Fahrzeuge mit Brennstoffzelle als Range Extender in vernetzten Verkehrssystemen zur Lösung der Problemstellung vorgestellt wird. Es wird vorausgesetzt, dass das Fahrzeug sich in einem digital vernetzten Verkehrssystem befindet, in dem Informationen über das aktuelle Verkehrsgeschehen und über den Status von Lade- und Tankinfrastruktur durch V2X-Kommunikation vorliegen.

In diesem Beitrag werden zunächst das methodische Vorgehen (Kap. 1) und der Stand des Wissens (Kap. 2) als Grundlage zur Konzeption der intelligenten Zielführung (Kap. 3) dargestellt. Daraufhin erfolgen die Modellierung des Straßennetzes und der Energiespeicher (Kap. 4) sowie die Auslegung der Zielführung (Kap. 5). Diese wird mittels Model-in-the-Loop-(MiL-), Software- 
in-the-Loop-(SiL-) und Hardware-in-the-Loop-(HiL)-Simulationen erprobt (Kap. 6). Abschließend wird ein Resümee gezogen und ein Ausblick gegeben (Kap. 7).

\section{Methodik}

Zur Entwicklung der intelligenten Zielführung wird nach dem mechatronischen Entwicklungskreislauf (Figure 1), einer Methodik zur durchgängig modellbasierten Entwicklung und -absicherung mechatronischer Systeme nach [1], vorgegangen. Die Methodik zeichnet sich durch Iterationsmöglichkeiten zu jedem Zeitpunkt aus, sodass Fehler frühzeitig behoben werden und Entwicklungszeit sowie Entwicklungskosten gesenkt werden können.

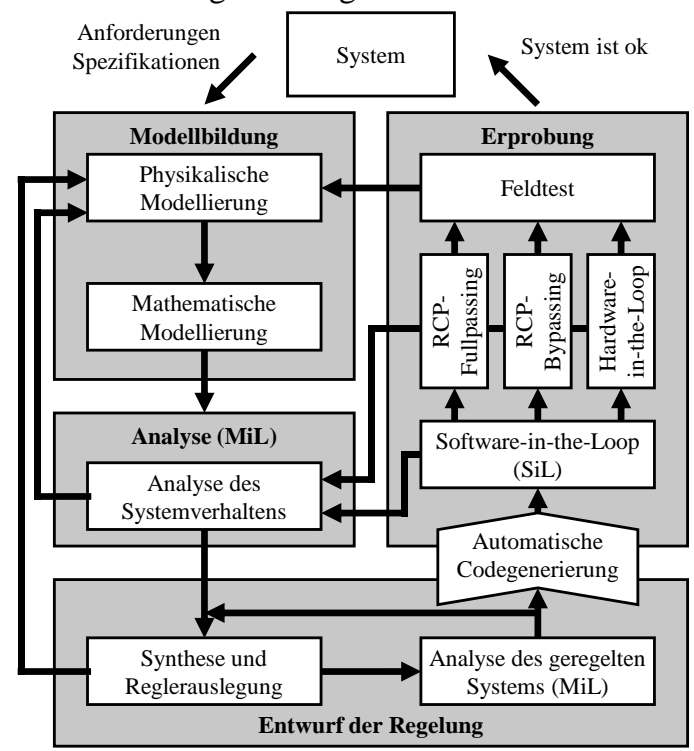

Figure 1: Mechatronischer Entwicklungskreislauf zur Entwicklung mechatronischer Systeme.

Der Entwicklungskreislauf beginnt mit der Definition von Anforderungen und Spezifikationen, die das System erfüllen muss. Mit diesen Kenntnissen wird ein physikalisches Modell des Systems aufgestellt, aus welchem ein mathematisches Modell abgeleitet wird. Die Modellparameter werden aus technischen Unterlagen oder Messungen an realen Komponenten ermittelt. Diesem Modellbildungsprozess folgt die Analyse des Systemverhaltens durch MiL-Simulationen und ggf. eine Änderung des zugrundeliegenden Modells. Nach erfolgreicher Analyse des Systemverhaltens erfolgt die Reglerauslegung mit der Reglersynthese und der Analyse des geregelten Systems ebenfalls durch MiL-Simulationen. Sobald das geregelte System erfolgreich analysiert wurde, erfolgt die automatisierte Codegenerierung des Reglers, sodass der
Regler ohne fehleranfällige manuelle Programmierung als Code vorliegt. Dieser Code wird in SiL-Simulationen abermals untersucht, bevor weitere Verifikationen und Optimierungen unter Echtzeitbedingungen im RCP-Fulloder Bypassing oder in HiL-Simulationen durchgeführt werden. Abschließend erfolgen Feldtests, die den mechatronischen Entwicklungskreislauf bei Erfüllung aller Anforderungen und Spezifikationen beenden.

\section{Stand des Wissens}

Dieses Kapitel stellt den Stand des Wissens zu Zielführungsverfahren, zur Integration dynamischer Informationen und zur Zielführung mit Zwischenzielen dar.

\subsection{Verfahren zur Zielführung}

Grundlage der Zielführung sind bewertete und gerichtete Graphen. Es existiert eine Vielzahl von Algorithmen zur Optimierung in Graphen mit unterschiedlichen Zielstellungen, von denen nur jene zum Auffinden optimaler Pfade zwischen zwei Knoten betrachtet werden.

Das bekannteste Verfahren zum Auffinden eines optimalen Pfades in einem bewerteten Graphen ist der von Dijkstra [2] vorgestellte Algorithmus. Es handelt sich um ein Verfahren der Breitensuche, welches immer die optimale Lösung bzgl. eines definierten Bewertungskriteriums findet, sofern der Graph keine negativen Kantenbewertungen beinhaltet. Nachteilig ist der hohe Rechenaufwand. Auf verwandte Algorithmen wie den BellmanFord-Algorithmus oder den Floyd-Warshall-Algorithmus soll nicht weiter eingegangen werden. Der DijkstraAlgorithmus bildet die Grundlage vieler Ansätze, durch die seine Effizienz deutlich gesteigert wird. Für einen Überblick über diese Ansätze sei auf [3] verwiesen.

Neben diesen analytischen Ansätzen existieren auch stochastische Ansätze wie die von [4] beschriebene Ant Colony Optimization (ACO). Das Verfahren arbeitet schneller als exakte Algorithmen, allerdings kann das Auffinden der optimalen Route nicht analytisch nachgewiesen werden. Auch Ansätze der Künstlichen Intelligenz und des Reinforcement Learnings können wie von [5] dargestellt zur Zielführung eingesetzt werden.

Es existiert eine Vielzahl von Anwendungen dieser Verfahren zur Zielführung elektrischer Fahrzeuge, welche den Energieverbrauch ohne Lade- und Tankstopps optimieren. Stellvertretend sei der von [6] fokussierte Ansatz zur energieoptimierten Zielführung eines Elektrofahrzeugs mit Batterie und Superkondensator genannt. 


\subsection{Integration dynamischer Informationen}

V2X-Nachrichten werden i.d.R. durch Anpassung des Graphen ähnlich wie Informationen aus dem Traffic Message Channel (TMC) verarbeitet. Diese können wie von [7] genutzt werden, um die eigene Zielführung zu optimieren und Staus zu umfahren, oder wie von [8] vorgeschlagen, um mithilfe der Zustandsdaten vieler Verkehrsteilnehmer den Verkehrsfluss zu optimieren und Stausituationen im Voraus zu vermeiden.

\subsection{Zielführung mit Zwischenzielen}

Ansätze der Tourenplanung, wie von [9] für Zustellfahrzeuge oder von [10] und [11] im Allgemeinen für logistische Systeme vorgestellt, ermitteln optimale Touren durch definierte Zwischenziele. Der Ansatz von [12] berücksichtigt die beschränkte Reichweite eines Elektronutzfahrzeugs bei der Tourenplanung, allerdings ohne weitere Ladestopps zu planen. In der Regel handelt es sich bei der Tourenplanung um Offline-Verfahren.

In [13] wird ein Ansatz zum Auffinden energieoptimaler Routen für batterieelektrische Fahrzeuge unter Berücksichtigung notwendiger Ladestopps dargestellt, bei dem es sich um eine Erweiterung eines Multi-LevelDijkstra-Verfahren handelt. Ziel ist die Minimierung der durch Ladung aufgenommenen Energie, um eine möglichst kurze Fahrtdauer zu erzielen. Auch Online-Rechner wie GoingElectric oder Apps wie Wattfinder und Next Plug schlagen Ladestopps vor, berücksichtigen allerdings ebenfalls keine Wasserstofftankstellen.

\subsection{Zwischenfazit}

Insgesamt lässt sich feststellen, dass eine Vielzahl von Ansätzen zur Zielführung existieren. Diese finden einen optimalen Pfad bzgl. eines definierten Bewertungskriteriums wie Fahrtdauer, Distanz oder Energieverbrauch, berücksichtigen aber keine Zwischenziele, sodass sie nicht zur Lösung der Problemstellung geeignet sind. Die im Bereich der Logistik verbreiteten Ansätze der Tourenplanung sind ebenfalls nicht geeignet, da nicht nur das Auffinden einer optimalen Route durch gegebene Zwischenziele, sondern auch die Wahl dieser Zwischenziele die Optimierungsaufgabe zur Lösung der Problemstellung darstellt. [13] stellt einen Ansatz zur Wahl dieser Zwischenziele für ein batterieelektrisches Fahrzeug dar, berücksichtigt allerdings nicht die Planung von Tankstopps für den zweiten Energieträger Wasserstoff.

Keiner der dargestellten Ansätze ist zur vollständigen Lösung der Problemstellung geeignet.

\section{Konzeption der intelligenten Zielführung}

In diesem Abschnitt werden Anforderungen an die intelligente Zielführung erhoben und ein Konzept der intelligenten Zielführung aufgestellt.

\subsection{Anforderungen}

1. Das Kartenmaterial des Verkehrssystems muss in einer geeigneten mathematischen Beschreibungsform vorliegen, um die Zielführung zu ermöglichen. Es muss Informationen über Fahrtdauer, Strecke, Höhenprofil, Energieverbrauch sowie Lade- und Tankinfrastruktur beinhalten, um diese bei der Zielführung berücksichtigen zu können.

2. Durch die Zielführung soll je nach Fahrer-/Insassenwunsch die kürzeste, schnellste oder energieeffizienteste Route zum Fahrtziel bestimmt werden.

3. Aktuelle Informationen über das Verkehrsgeschehen und die Verfügbarkeit von Infrastruktur aus der V2XKommunikation sollen berücksichtigt werden können.

4. Durch die Zielführung müssen bei Bedarf Lade- und Tankstopps geplant werden, um das Fahrtziel erreichen zu können. Diese Planung soll drei Betriebsmodi (Batteriebetrieb, Brennstoffzellenbetrieb und leistungsverteilten Betrieb) ermöglichen.

5. Für den leistungsverteilten Betrieb muss die Zielführung Lade- und Tankstopps derart planen, dass sowohl Batterie als auch Wasserstoffspeicher immer über ausreichend Energie verfügen, um beide Energiequellen im Wirkungsgradoptimum betreiben zu können.

\subsection{Konzept der intelligenten Zielführung}

In Figure 2 ist das Konzept der intelligenten Zielführung als Funktionsstruktur dargestellt. Kern der Zielführung ist das statische Kartenmaterial, welches in geeigneter Form bereitgestellt werden muss. Es wird das frei zugängliche Kartenmaterial der OpenStreetMap [14] vorgesehen, welchem Höhendaten und verlässliche Daten über Ladesäulen und Wasserstofftankstellen hinzugefügt werden. Die Informationen aus der Ermittlung von Fahrzeugzuständen, der Umfeldperzeption und der V2XKommunikation mit anderen Verkehrsteilnehmern sowie der Infrastruktur werden genutzt, um das statische Kartenmaterial zu aktualisieren und es um dynamische Informationen zu erweitern. Des Weiteren werden GPS-Daten aus der Zustandserfassung sowie die Ergebnisse der Umfeldperzeption in SLAM-Verfahren genutzt, um die 
Selbstlokalisierung des Ego-Fahrzeugs durchzuführen, welche den Startpunkt der Zielführung darstellt. Basierend auf der dynamischen Karte, der Ego-Position und dem Fahrtziel wird durch die Zielführung, unter Berücksichtigung eines gewählten Betriebsmodus, eine bzgl. Fahrtdauer, Distanz oder Energieverbrauch optimierte Route ermittelt. Etwaige notwendige Lade- oder Tankstopp werden ebenfalls durch die Zielführung geplant.

In diesem Beitrag werden aus der Funktionsstruktur die Bereitstellung des Kartenmaterials (Kap. 4.1 bis 4.3), die Kartenaktualisierung durch V2X-Informationen (Kap. 5.1) sowie die Zielführung (Kap. 5.2 und 5.3) betrachtet.

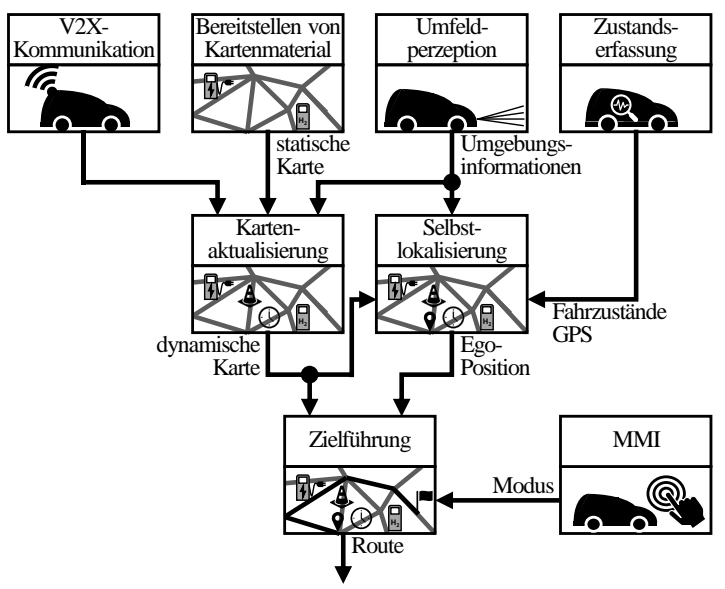

Figure 2: Funktionsstruktur der intelligenten Zielführung.

\section{Modellbildung}

Dieses Kapitel beschreibt die Modellbildung des Straßennetzes als Datengrundlage der Zielführung und die Modellierung der Energiespeicher, welche zur Planung der Lade- und Tankstopps benötigt werden.

\subsection{Beschreibung des Straßennetzes}

Das Straßennetz wird als Graph $G(N, E)$ beschrieben, welcher nach [15] aus einer Menge $N$ von Knoten $n_{\mathrm{i}}$ und einer Menge $E$ von Kanten $e_{\mathrm{a}, \mathrm{b}}$, die jeweils zwei Knoten $n_{\mathrm{a}}$ und $n_{\mathrm{b}}$ miteinander verbinden, besteht. Die Kanten des Graphen werden sowohl gerichtet als auch bewertet, da durch die Richtung der Kanten einerseits die Berücksichtigung von Einbahnstraßen oder Sperrungen einzelner Fahrtrichtungen und durch die Bewertung der Kanten andererseits die Berücksichtigung von Straßenlänge, Fahrtdauer oder Energieverbrauch ermöglicht wird.

Dem Graphen des Straßennetzes werden die frei zugänglichen Höhendaten der NASA aus der Shuttle Radar Topography Mission (SRTM, [16]) überlagert, um den
Einfluss der Fahrbahnneigung auf den Energieverbrauch abbilden zu können. Zur Veranschaulichung des aus der OSM abgeleiteten Graphen mit überlagerten SRTM-Daten ist dieser im Umfeld des Hauptcampus der Ostfalia Hochschule in Wolfenbüttel in Figure 3 dargestellt.

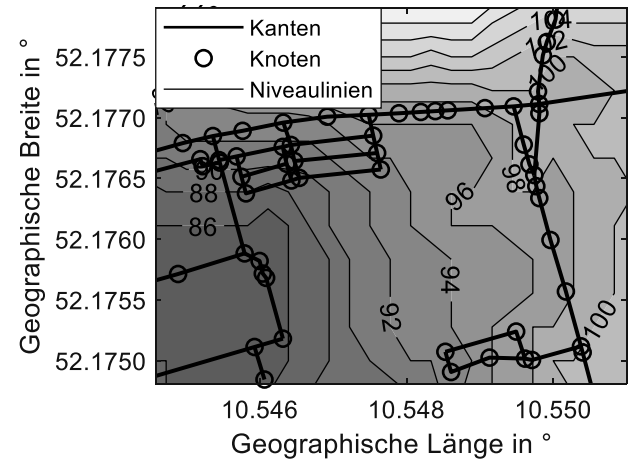

Figure 3: Überlagerung von Graph und Höhenprofil an der Ostfalia in Wolfenbüttel.

\subsection{Bewertung des Graphen}

Die Kanten des Graphen werden mit der Distanz $s$, der Dauer $t$ und der Energie $E$ bewertet, welche zum Übergang eines Knotens $a$ zum Knoten $b$ anfallen. Jeder Kante $e_{\mathrm{a}, \mathrm{b}}$ wird entsprechend eine Bewertung

$$
\underline{w}_{\mathrm{a}, \mathrm{b}}=\left[\begin{array}{lll}
s_{\mathrm{a}, \mathrm{b}} & t_{\mathrm{a}, \mathrm{b}} & E_{\mathrm{a}, \mathrm{b}}
\end{array}\right]^{T}
$$

zugewiesen. Die Distanz

$$
s_{\mathrm{a}, \mathrm{b}}=2 r \cdot \arcsin \left(\sqrt{c_{\mathrm{a}, \mathrm{b}}}\right)
$$

mit

$$
c_{\mathrm{a}, \mathrm{b}}=\sin ^{2}\left(\frac{\Delta \varphi_{\mathrm{a}, \mathrm{b}}}{2}\right)+\cos \left(\varphi_{\mathrm{a}}\right) \cos \left(\varphi_{\mathrm{b}}\right) \sin ^{2}\left(\frac{\Delta \lambda_{\mathrm{a}, \mathrm{b}}}{2}\right)
$$

wird nach den von [17] vorgestellten Gleichungen (2) und (3) mithilfe der Längengradsdifferenz $\Delta \lambda_{\mathrm{a}, \mathrm{b}}$, der Breitengrade $\varphi_{\mathrm{a}}$ und $\varphi_{\mathrm{b}}$, der Breitengradsdifferenz $\Delta \varphi_{\mathrm{a}, \mathrm{b}}$ sowie dem Erdradius $r$ berechnet. Die Dauer

$$
t_{\mathrm{a}, \mathrm{b}}=t_{\mathrm{min}, \mathrm{a}, \mathrm{b}}+t_{\mathrm{B}, \mathrm{a}, \mathrm{b}}+t_{\mathrm{W}, \mathrm{a}, \mathrm{b}}+t_{\mathrm{V}, \mathrm{a}, \mathrm{b}}
$$

basiert u. a. auf der minimalen Fahrtdauer $t_{\text {min,a,b }}$, die sich für die Fahrt mit der zulässigen Höchstgeschwindigkeit ergibt. Des Weiteren werden Zeitaufschläge für Beschleunigungs- und Bremsvorgänge $t_{\mathrm{B}, \mathrm{a}, \mathrm{b}}$, für Wartezeiten $t_{\mathrm{W}, \mathrm{a}, \mathrm{b}}$, bspw. an Kreuzungen oder Lichtsignalanlagen, sowie für verkehrsbedingtes Unterschreiten der Höchstgeschwindigkeit $t_{\mathrm{V}, \mathrm{a}, \mathrm{b}}$ berücksichtigt. Die Energie

$$
E_{\mathrm{a}, \mathrm{b}}=f\left(v_{\mathrm{a}, \mathrm{b}}, a_{\mathrm{a}, \mathrm{b}}, \alpha_{\mathrm{a}, \mathrm{b}}\right)+P_{\mathrm{BN}} \cdot t_{\mathrm{a}, \mathrm{b}}
$$

wird mithilfe eines inversen Fahrzeug- und Antriebsstrangmodells sowie dem Leistungsbedarf des Bordnetzes $P_{\mathrm{BN}}$ und der Dauer $t_{\mathrm{a}, \mathrm{b}}$ abgeschätzt. Wesentliche Einflussgrößen sind die prognostizierte Geschwindigkeit 
$v_{\mathrm{a}, \mathrm{b}}$, welche verkehrsbedingt nicht zwingend der Höchstgeschwindigkeit entspricht, die Fahrzeugbeschleunigung $a_{\mathrm{a}, \mathrm{b}}$ sowie die Fahrbahnsteigung $\alpha_{\mathrm{a}, \mathrm{b}}$.

\subsection{Lade- und Tankinfrastruktur}

Informationen über die Ladesäulen- und Wasserstofftankstelleninfrastruktur der OSM sind nicht zuverlässig, da sie ohne Qualitätskontrolle von Nutzern gepflegt werden. Für die Zielführung allerdings sind aktuelle Informationen über die Infrastruktur elementar, da fehlerhafte Informationen zum Ausbleiben notwendiger Lade- bzw. Tankstopps und somit zum Liegenbleiben des Fahrzeugs führen können. Aus diesem Grund werden die Ladesäulen der von der Bundesnetzagentur gepflegten Ladesäulenkarte [18] entnommen. Diese enthält deutschlandweit alle Ladesäulen, welche nach der Ladesäulenverordnung als öffentlich zugänglich gemeldet sind. Informationen über die europaweite, vergleichsweise sehr überschaubare, Wasserstofftankinfrastruktur werden von H2 Mobility bezogen [19].

Die Integration der Infrastrukturdaten erfolgt durch Zuordnung zu entsprechenden Knoten des Graphen. Des Weiteren werden diese Knoten und der Status der Infrastruktur in einer Infrastrukturliste eingetragen, welche als Grundlage der infrastrukturoptimierten Zielführung (vgl. Kap. 5.3) dient. Das Ergebnis der Integration der Infrastruktur in den Graphen ist in Figure 4 dargestellt. Der Übersichtlichkeit halber ist nur das Straßennetz der deutschen Bundesautobahn (BAB) mit der nahe der BAB gelegenen Lade- und Tankinfrastruktur eingezeichnet.

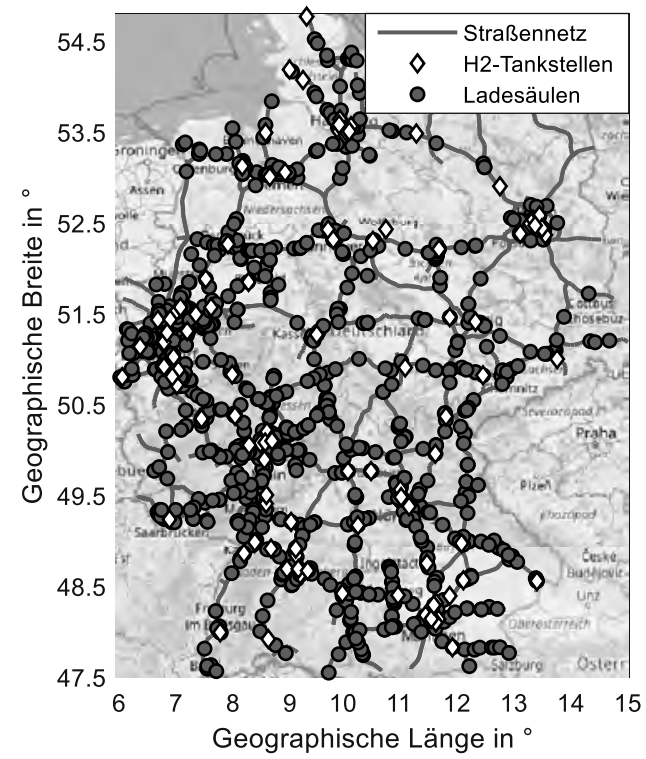

Figure 4: Straßennetz der BAB mit nahegelegener Ladeund Tankinfrastruktur.

\subsection{Modellierung der Energiespeicher}

Wie in der Motivation beschrieben, kann die Fahrzeugleistung durch eine Batterie und eine Brennstoffzelle aufgebracht werden, sodass sich die für die Fahrt aufgebrachte Energie für den Übergang von Knoten $a$ zu $b$

$$
E_{\mathrm{a}, \mathrm{b}}=E_{\mathrm{bat}, \mathrm{a}, \mathrm{b}}+E_{\mathrm{bz}, \mathrm{a}, \mathrm{b}}
$$

aus der Energie der Batterie $E_{\mathrm{bat}, \mathrm{a}, \mathrm{b}}$ und der Brennstoffzelle $E_{\mathrm{bz}, \mathrm{a}, \mathrm{b}}$ zusammensetzt. Mithilfe des Faktors

$$
k_{\text {mod }}=\left\{\begin{array}{cc}
0 & \text { für Brennstoffzellenbetrieb } \\
10,1[ & \text { für leistungsverteilten Betrieb } \\
1 & \text { für Batteriebetrieb }
\end{array}\right.
$$

werden die Energie von Batterie

$$
E_{\mathrm{bat}, \mathrm{a}, \mathrm{b}}=k_{\text {mod }} E_{\mathrm{a}, \mathrm{b}}
$$

und Brennstoffzelle

$$
E_{\mathrm{bz}, \mathrm{a}, \mathrm{b}}=\left(1-k_{\mathrm{mod}}\right) E_{\mathrm{a}, \mathrm{b}}
$$

aus der Gesamtenergie berechnet. Zur Abschätzung der Ladestandsänderung der Batterie und der Massenänderung im Wasserstofftank wird vereinfachend angenommen, dass sowohl Batterie als auch Brennstoffzelle konstant mit Nennspannung betrieben werden. Der Ladestand am Knoten $b$

$$
S O C_{\mathrm{bat}, \mathrm{b}} \cong S O C_{\mathrm{bat}, \mathrm{a}}+\frac{E_{\mathrm{bat}, \mathrm{a}, \mathrm{b}}}{\eta_{\mathrm{bat}} C_{\mathrm{bat}}}
$$

ergibt sich somit in Abhängigkeit des Startladestandes $S O C_{\mathrm{a}}$, der Energie $E_{\mathrm{bat}, \mathrm{a}, \mathrm{b}}$, des Coloumb'schen Wirkungsgrades $\eta_{\text {bat }}$ sowie der Nennkapazität $C_{\text {bat }}$. Analog ergibt sich die vorhandene Wasserstoffmasse

$$
m_{\mathrm{H}_{2}, \mathrm{~b}} \cong m_{\mathrm{H}_{2}, \mathrm{a}}+a_{\mathrm{bz}} \frac{E_{\mathrm{bz}, \mathrm{a}, \mathrm{b}}}{u_{\mathrm{bz}}}
$$

abhängig von der Energie $E_{\mathrm{bz}, \mathrm{a}, \mathrm{b}}$, der Nennspannung $u_{\mathrm{bz}}$ sowie der Konstante $a_{\mathrm{bz}}$, welche den Massebedarf nach dem Faraday'schen Gesetz sowie die Zellanzahl des Brennstoffzellenstacks und die Stöchiometrie beinhaltet.

\section{Auslegung der Zielführung}

Dieses Kapitel stellt die Auslegung der Zielführung vor.

\subsection{Integration dynamischer Informationen}

Die Einbindung dynamischer Informationen aus der V2X-Kommunikation wird durch eine temporäre Anpassung der Kantenbewertungen im Graphen oder eine Statusaktualisierung in der Infrastrukturliste realisiert.

Zur Anpassung der Kantenbewertungen wird aus der GPS-Position der V2X-Nachricht zunächst ermittelt, 
welcher Kante die Informationen zuzuweisen ist. Anschließend erfolgt die Änderung der Kantengewichte je nach Art der Information. Tritt bspw. eine Straßensperrung infolge einer Baustelle auf, werden für die entsprechende Kante sowohl Distanz als auch Dauer und Energieverbrauch auf unendlich gesetzt, sodass die Kante unabhängig der Gewichtungsfaktoren nicht mehr Bestandteil der optimalen Route sein kann. Tritt hingegen eine Verzögerung durch stockenden Verkehr auf, werden nur Dauer und Energieverbrauch der Kante geändert, da sich die Streckenlänge nicht ändert.

\subsection{Optimale Zielführung}

Der grundlegende Algorithmus zur optimalen Zielführung sucht einen optimalen Pfad durch den Graphen des Straßennetzes vom Start- zum Zielknoten. Als Optimierungsverfahren zur Bestimmung dieses optimalen Pfades wird der Suchalgorithmus nach Dijkstra gewählt. Mithilfe der Kostenfunktion

$$
J_{\mathrm{b}}\left(J_{\mathrm{a}}, \underline{w}_{\mathrm{a}, \mathrm{b}}\right)=J_{\mathrm{a}}+\left[\begin{array}{lll}
g_{\mathrm{s}} & g_{\mathrm{t}} & g_{\mathrm{E}}
\end{array} \underline{w}_{\mathrm{a}, \mathrm{b}}\right.
$$

werden die Kosten $J_{\mathrm{b}}$ nach einem Übergang von Knoten a zu Knoten b abhängig von den Kosten $J_{\mathrm{a}}$ und der Kantenbewertung $\underline{w}_{\mathrm{a}, \mathrm{b}}$ bestimmt. Mithilfe der Gewichtungsfaktoren $g_{\mathrm{s}}, g_{t}$ und $g_{\mathrm{E}}$ wird definiert, ob die Route bzgl. der Distanz, der Fahrtdauer, des Energieverbrauchs oder eines Kompromisses dieser Kriterien optimiert werden soll. Die Gesamtkosten jedes Knotens werden mit Start des Algorithmus bis auf Ausnahme des Startknotens, welcher mit null initialisiert wird, mit unendlich initialisiert. Der Algorithmus bestimmt ausgehend vom Startknoten die Kosten der unbesuchten Nachbarknoten nach Gl. 12 und aktualisiert die Gesamtkosten der Nachbarknoten, wenn die neu berechneten Kosten kleiner als die vorherigen Kosten sind. Alle betrachteten Nachbarknoten werden in eine Warteliste aufgenommen, der Startknoten wird als besucht markiert und der Knoten mit den geringsten Kosten wird aus der Warteliste ausgewählt. Von diesem Knoten ausgehend wiederholt sich das beschriebene Vorgehen, bis der Zielknoten erreicht und der optimale Pfad ermittelt wurde.

\subsection{Infrastrukturoptimierte Zielführung}

Die infrastrukturoptimierte Zielführung wird relevant, wenn das Fahrtziel nicht ohne Lade- oder Tankstopps erreicht werden kann.

In Figure 5 ist der Programmablaufplan (PAP) der infrastrukturoptimierten Zielführung für alle Betriebsmodi dargestellt. Zunächst erfolgt eine Aktualisierung des Kartenmaterials und der Infrastrukturliste durch V2X-Informationen, bevor die in Kap. 5.2 vorgestellte Zielführung vom Start zum Ziel durchgeführt wird. Das Ziel kann erreicht werden, wenn im Batteriebetrieb der Ladestand am Ziel größer als null ist, im Brennstoffzellenbetrieb die verfügbare Wasserstoffmasse im Tank am Ziel größer als null ist und im leistungsverteilten Betrieb sowohl der Ladestand als auch die verfügbare Wasserstoffmasse im Tank am Ziel größer als null sind.

Sind diese Bedingungen nicht erfüllt, kann das Ziel nicht ohne zusätzliche Energieaufnahme erreicht werden. Deshalb wird zunächst die unbesuchte Lade- und Tankinfrastruktur innerhalb der Reichweite ermittelt. Zur Reduktion der Menge an Ladesäulen und Tankstellen werden nur diejenigen betrachtet, an denen Restladestand bzw. Restwasserstoffmasse definierte Grenzwerte unterschreiten, sodass eine möglichst hohe Energieaufnahme erzielt wird. Die übrig gebliebene Menge der Lade- und Tankinfrastruktur wird, wenn nicht bereits geschehen, in eine Warteliste eingetragen. Hierbei wird den Kosten ein Zeitaufschlag für den Lade- oder Tankvorgang hinzugefügt. Aus der Warteliste wird ein Lade- oder Tankstopp als neuer Startpunkt ausgewählt, von dem aus die Zielführung zum ursprünglichen Ziel erfolgt. Dieses Prozedere wiederholt sich bis die Warteliste leer ist. Abschließend wird die beste Route bzgl. eines definierten Zielkriteriums (vgl. Gl. 12) ausgewählt.

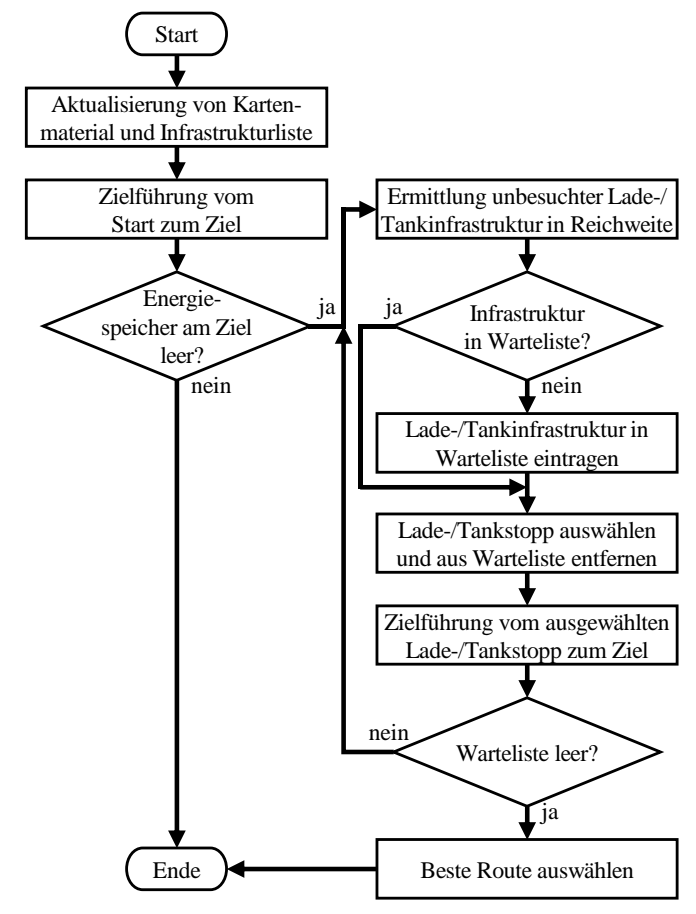

Figure 5: PAP der infrastrukturoptimierten Zielführung zur Planung von Tank- und Ladestopps. 


\section{Erprobung}

Dieses Kapitel stellt exemplarische Ergebnisse der Erprobung in MiL-, SiL- und HiL-Simulationen vor.

\subsection{MiL- und SiL-Simulationen}

In diesem Abschnitt werden offline erzielte Ergebnisse aus MiL- und SiL-Simulationen vorgestellt.

In Figure 6 sind die infrastrukturoptimierten Routen von Hamburg nach München für die drei Betriebsmodi mit geplanten Lade- und Tankstopps für ein Fahrzeug mit einer Batteriekapazität von 42 kWh und einem Speichervermögen von 2,5 kg Wasserstoff dargestellt.

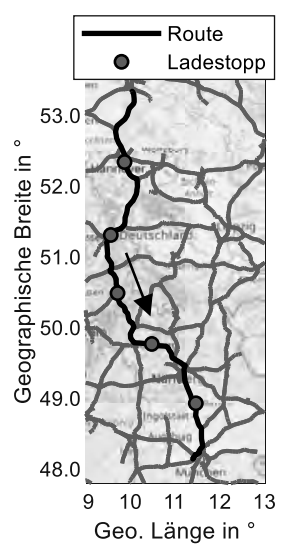

a) Batteriebetrieb

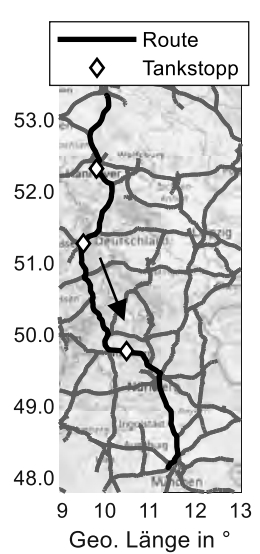

b) Brennstoffzellenbetrieb

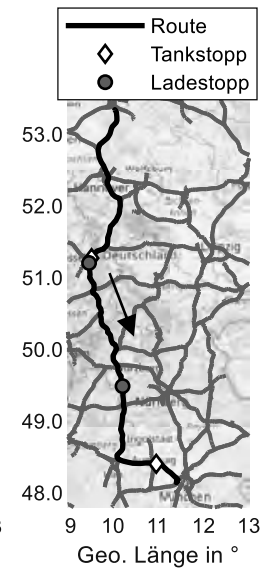

c) Leistungsverteilter Betrieb
Figure 6: Gegenüberstellung infrastrukturoptimierter Routen von Hamburg nach München für den Batteriebetrieb (a), den Brennstoffzellenbetrieb (b) und den leistungsverteilten Betrieb (c).

Im Batteriebetrieb wurde eine Strecke von $761 \mathrm{~km}$ in insgesamt 11,2 h, im Brennstoffzellenbetrieb eine Strecke von $770 \mathrm{~km}$ in 8,1 h und im leistungsverteilten Betrieb eine Strecke von 790 km in 9,4 h zurückgelegt.

Im Batteriebetrieb ergibt sich erwartungsgemäß aufgrund der langen Ladezeiten die längste Fahrtdauer, allerdings kann aufgrund der verhältnismäßig hohen Anzahl an Ladesäulen die kürzeste Strecke ohne Umwege zurückgelegt werden. Im Brennstoffzellenbetrieb wird die gleiche Route wie im Batteriebetrieb gewählt, allerdings werden aufgrund der geringen Anzahl an Wasserstofftankstellen kleine Umwege zu diesen nötig. Die Fahrtdauer ist am geringsten, da der Tankvorgang deutlich schneller als das Laden vonstattengeht. Im leistungsverteilten Betrieb resultiert die Fahrzeit aus der Kombi- nation schneller Tankvorgänge mit langsameren Ladevorgängen. Insgesamt wurde in diesem Modus eine längere Strecke zurückgelegt, allerdings wurden die Energieverluste in der Leistungsbereitstellung um 52,81\% verglichen zum Batteriebetrieb und um 44,59 \% verglichen zum Brennstoffzellenbetrieb reduziert. Weitere Informationen zu Einsparpotentialen durch den leistungsverteilten Betrieb können [20] entnommen werden.

\subsection{HiL-Simulation}

Mithilfe eines mobilen HiL-Prüfstandes (vgl. [21]) wurde die Zielführung unter Einbezug dynamischer Informationen aus der V2X-Kommunikation online unter Echtzeitbedingungen mit realer Hardware getestet.

Die Versuche wurden ohne und mit Störung durch eine Baustelle auf dem Innenhof der Ostfalia Hochschule in Wolfenbüttel durchgeführt. Die Ergebnisse sind in Figure 7 dargestellt. Es ist offensichtlich, dass die Route ohne Baustelle die optimale Route ist. Es lässt sich feststellen, dass die Zielführung in der Echtzeitumgebung des mobilen HiL-Prüfstands korrekt funktioniert.

Daraufhin wird eine V2X-Nachricht empfangen, welche eine Sperrung der optimalen Route durch eine Baustelle mitteilt. Die Zielführung verarbeitet diese Information und wählt die kürzeste Alternativroute. Dieses trivial wirkende Ergebnis zeigt, dass zum einen die V2X-Kommunikation und zum anderen die Integration der V2XNachricht in die Zielführung (vgl. Kap. 5.1) funktioniert und eine Neuberechnung der Route einleitet.

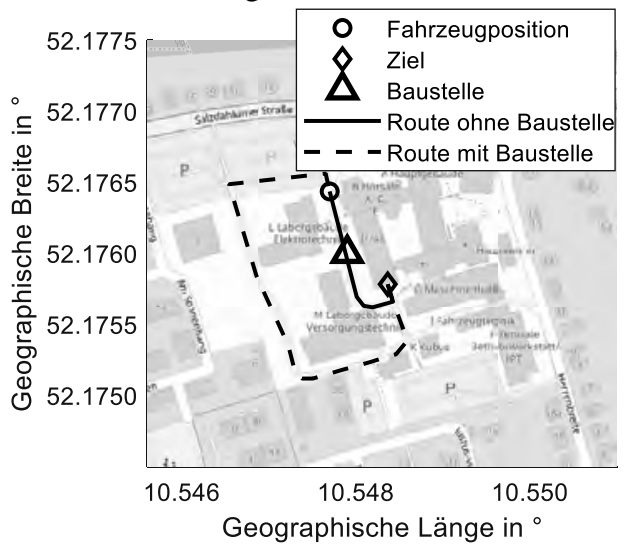

Figure 7: Ergebnisse der Online-Zielführung unter Echtzeitbedingungen auf dem Innenhof der Ostfalia in Wolfenbüttel.

\section{Resümee und Ausblick}

Dieser Beitrag stellt die Auslegung einer intelligenten Zielführung für Elektrofahrzeuge mit Brennstoffzelle als 
Range Extender in vernetzten Verkehrssystemen dar.

Es wurde eine Schnittstelle zur Integration dynamischer Informationen aus der V2X-Kommunikation vorgesehen, durch welche das Kartenmaterial stetig aktualisiert wird. Die Zielführung ermöglicht einen optimierten Betrieb bzgl. Fahrtdauer, Fahrtstrecke, Energieverbrauch und einem Kompromiss dieser Kriterien. Je nach Betriebsmodus (Batteriebetrieb, Brennstoffzellenbetrieb, Leistungsverteilter Betrieb) werden notwendige Ladeoder Tankstopps eingeplant, sollte die Energie zum Erreichen des Ziels nicht ausreichen. Die komplette Zielführung wurde offline im Rahmen von MiL- und SiL-Simulationen erprobt. Die V2X-Schnittstelle und die Zielführung ohne Berücksichtigung der Infrastruktur wurden des Weiteren online in einer HiL-Simulation unter Echtzeitbedingungen erprobt.

Zukünftige Arbeitsschwerpunkte liegen zum einen in weiteren Untersuchungen und Optimierungen mittels MiL-, SiL- und HiL-Simulationen und zum anderen in der Realisierung der infrastrukturoptimierten Zielführung als online-Verfahren in einer Echtzeitumgebung. Hierzu werden Optimierungspotentiale in der Reduktion des zugrundeliegenden Graphen des Straßennetzes, in Maßnahmen zur Effizienzsteigerung des Dijkstra-Algorithmus zur Zielführung und in einer Reduktion der Zielführungsaufrufe durch die infrastrukturoptimierte Zielführung untersucht.

\section{Danksagung}

Dieser Beitrag wurde im Rahmen des Verbundprojekts Zukünftige Fahrzeugtechnologien im Open Region Lab durch das Niedersächsische Ministerium für Wissenschaft und Kultur sowie die VolkswagenStiftung unter dem Förderkennzeichen VWZN3236 gefördert.

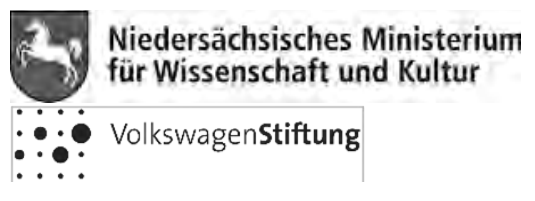

\section{Referenzen}

[1] Liu-Henke X. Mechatronische Entwicklung der aktiven Feder-/Neigetechnik für das Schienenfahrzeug RailCab. VDI-Fortschritt-Berichte, Reihe 12, Nr. 589, VDI-Verlag, Düsseldorf, Germany, 2004.

[2] Dijkstra E W. A Note on Two Problems in Connexion with Graphs. Numerische Mathematik. S. 269-271, 1959.

[3] Bast $\mathrm{H}$ et al. Route Planning in Transportation Networks, Lecture Notes in Computer Science, Vol. 9220,
S. 19-80, 2016.

[4] Dorigo M et al. Ant algorithms for discrete optimization. Artificial Life, Vol. 5, Nr. 2, S. 137-172, 1999.

[5] Yu J, Yu W, Gu J. Online Vehicle Routing With Neural Combinatorial Optimization and Deep Reinforcement Learning. IEEE Transactions on Intelligent Transportation Systems, Vol. 20, Nr. 10, October 2019.

[6] Jurik T et al. Energy Optimal Real-Time Navigation System. IEEE Intelligent Transportation Systems Magazine Vol. 6, Nr. 3, S. 66-79, 2014.

[7] Wang J et al. Dynamic Route Choice Prediction Model Based on Connected Vehicle Guidance Characteristics. Journal of Advanced Transportation, 2017.

[8] Backfrieder C et al. Increased Traffic Flow Thorugh Node-Based Bottleneck Prediction and V2X Communication. IEEE Transactions on Intelligent Transportation Systems, Vol. 18, Nr. 2, S. 349-363, February, 2017.

[9] Yu J, Yu W, Gu J. Online Vehicle Routing With Neural Combinatorial Optimization and Deep Reinforcement Learning. IEEE Transactions on Intelligent Transportation Systems, Vol. 20, Nr. 10, October, 2019.

[10] Fleischmann B, Kopfer H. Transport- und Tourenplanung. In: Tempelmeier H. Planung logistischer Systeme. Springer Vieweg, Berlin, 2018.

[11] Wenger W. Multikriterielle Tourenplanung. Dissertation, Universität Hohenheim. Gabler, Wiesbaden, 2010.

[12] Witte C, Marner, T. Tourenplanung mit Elektronutzfahrzeugen - ein GAMS-Modell. In Proff H, Fojcik T. Nationale und internationale Trends in der Mobilität. Springer Gabler, Wiesbaden, 2016.

[13] Baum M et al. Energy-Optimal Routes for Battery Electric Vehicles. Algorithmica, Vol. 82, Nr. 5, S. 14901546, 2020.

[14] OpenStreetMap Foundation. OpenStreetMap. Stand: 06.09.2020. https://www.openstreetmap.org

[15] Turau V, Weyer C. Algorithmische Graphentheorie. Auflage, De Gruyter, 2015.

[16] Farr T G. The Shuttle Radar Topography Mission. Reviews of Geophysics, 45, RG2004, 2007.

[17] Gade K. Non-singular Horizontal Position Representation. Journal of Navigation, Vol. 63, Nr. 3, S. 395-417, 2010.

[18] Bundesnetzagentur. Ladesäulenkarte. Stand: 07.08.2020.

[19] H2 Mobility Deutschland GmbH und Co. KG. H2.live. Stand: 06.09.2020. https://h2.live/tankstellen

[20] Scherler S et al. Predictive Energy Management for an Electric Vehicle with Fuel Cell Range Extender in Connected Traffic Systems. 19th IEEE Mechatronika, Prague, Czech Republic, December 2 - 4, 2020. (tbp)

[21] Scherler S, Liu-Henke X. Conception and Realization of a Mobile HiL Test Bench for V2X Communication. IEEE 91st Vehicular Technology Conference, Antwerp, Belgium, May 25-28, 2020. 